\title{
Heterosis and Combining Ability for Grain Yield and Yield Associated Traits in 10 X 10 Diallel Analysis in Pea (Pisum sativum L.)
}

\author{
Hariom Suman, Bijendra Kumar*, Nageshwar, Meenakshi Rathi and Dattesh Tamatam \\ Department of Genetics and Plant Breeding, School of Agriculture Lovely Professional \\ University, Phagwara, Punjab 144411, India \\ *Corresponding author
}

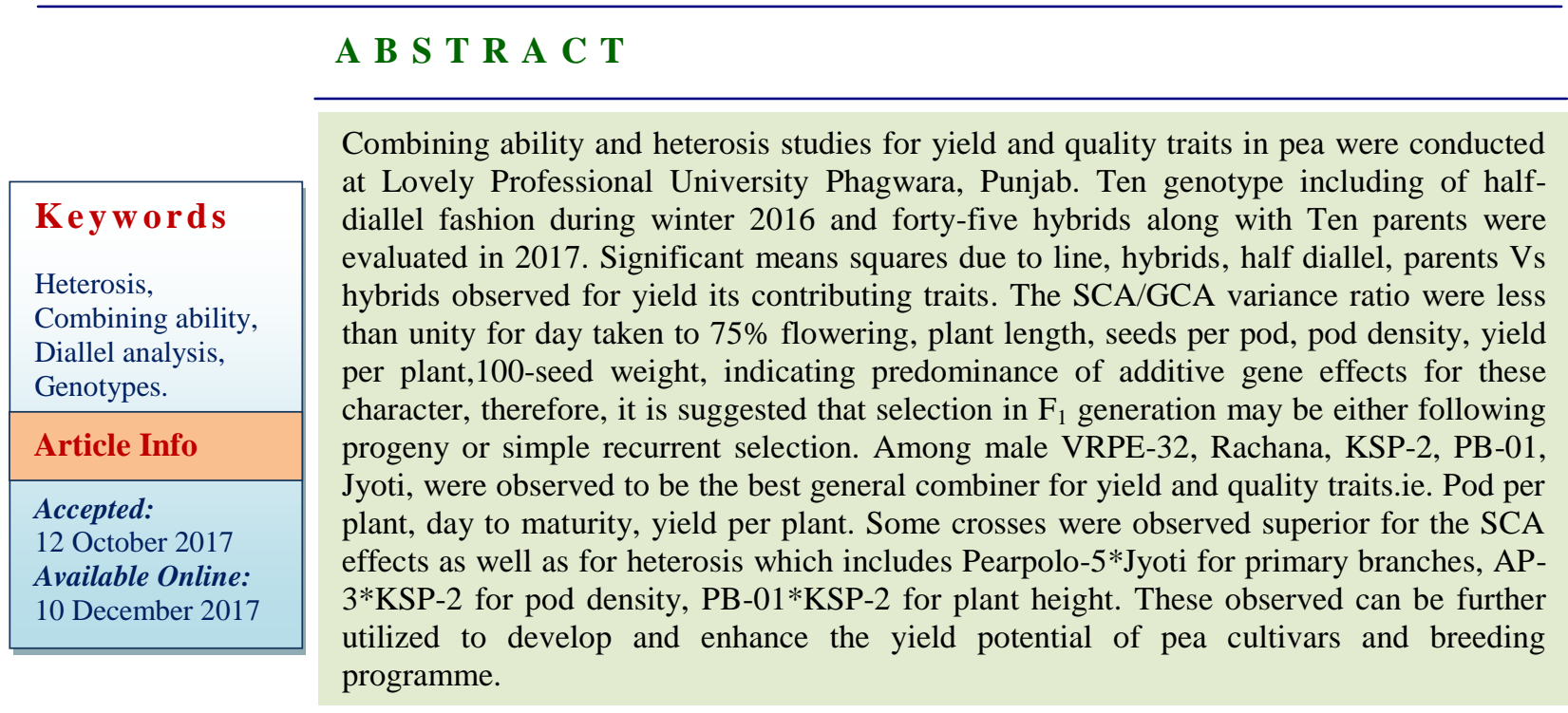

\section{Introduction}

Pea (Pisum sativum L.) is an important legume grown as a garden and field crop throughout the temperate region of the world; it is also grown as a cool season crop. It ranks third in production among the grain legumes after soybeans and beans in the worlds. Pea is the valued primarily for the nutritional quality having high protein as $20-30 \%$, vitamin A $5 \%$, beta carotene $4 \%$, and sufficient carbohydrates. The growth and development of peas are determined by the interaction of genetic factors, the environment and agricultural practices (Acikgoz et al., 2009). An improvement in yield of self-pollinated crop like garden pea is effected of mainly through selection of genotypes with superior characters for variation. Through recombination followed by selection heterosis in $\mathrm{F}_{1}$ generation as heterosis crosses may give a segregating and transgressive for economic traits in move forward generation. Breeding for superior varieties requires selection of parents capable of transmitting their desirable qualities. A rational approach for breeding is to select parents based on their combining ability rather than visual observations of their traits. The concept of combining ability analysis has significant practical importance 
in plant breeding (Tyagi and Srivastava, 2001). Precious steps for operation of heterosis in any crop are to study the GCA of the parents and SCA of hybrid. Ability of parents to combine well depends on the complex interaction among genes and its cannot to be estimated by yield performance of the parents. There the knowledge of combining ability and nature(environment) of gene effects is necessary for the selection of best parents hybridization in order to improve the existing cultivars, Although some information on a additive and non-additive effects of associated with yield and yield contributing traits in garden pea is available but that relevant the specific region, genetics material involved and particular environment conditions. India is the second largest producer of vegetables, next only to China, in the world with a production of 40 million tonnes from four million hectares of land area. In spite of that, this seemingly high level of production can provide only 208 grams of vegetables per capita (Sharma, 2003), as against the suggested dietary intake of $275 \mathrm{~g}$ and $250 \mathrm{~g}$ per capita per day for adult male and female, respectively for undertaking moderate work (Swaminathan, 2002). There for the presents investigated were carried out to obtain information regarding general and specific combining ability effects and finding of out heterosis combinations for yield and yields quality traits in garden pea.

\section{Materials and Methods}

The presented investigation was carried out at the Department of Plant Breeding and Genetics Lovely Professional University Phagwara, Punjab, during 2016-17. The experiment materials consist of garden pea, ten genotype viz., Pearpolo-5, vrpe-32, vrpe24, Jyoti, Rachana, KSP-2, 1-116, Sonali-10, PB-01, AP-3, were selected as lines for halfdiallel crosses. All the parents along with their $45^{\prime} F_{1}$ ' were grown in a random block design with three replications. The distance between the parents was maintained at $18 \mathrm{~cm}$ while the rows were spaced $25 \mathrm{~cm}$ apart. The standard plant protection and others cultivars practices were followed to maintain uniform experiments conditions. The analysis of variance was done for all character as per method given by Hayman (1954). Heterosis was works out over better parents was (heterobeltiosis) and standard check (standard heterosis) by Kempthorne (1957). Quantitative data were collected on five plants in each plot. Days to $75 \%$ flowering plant height, number of primary branches, pods/plant, pod length $(\mathrm{cm})$, pod density, number of seeds per pod, days to maturity, 100 -seed and yield/plant were recorded. Regarding the statistical analysis data recorded on parents and the $F_{1}$ hybrids were analyzed together as suggested by Singh and Chaudhary (1979). The combining ability analysis was done by Kempthorne (1957), which statistical analysis was applied also give references.

\section{Results and Discussion}

\section{Heterosis}

Heterosis is the superiority of $F_{1}$ over its parent. Heterosis was calculated in per cent over better parent on $F_{1}$ generation for all the 10 characters. Estimates of heterosis are presented in Table 8. Negative and significant values of heterosis were considered desirable for days to $75 \%$ flowering, plant height and days to maturity and on the other hand positive and significant values were considered desirable for other characters. The extent of heterosis over better parent for days to $75 \%$ flowering ranged from -2.03 to -16.43 per cent showing desirable heterosis for 44 hybrids (Table 5) out of 45 exhibited significant and desirable hybrids. Heterosis over better parent for plant height varied from -0.67 to -21.42 per cent showing desirable 
heterosis for 37 hybrids. The primary branches per plant are associated with positively desirable and significant. Significant heterosis was observed in six hybrids out of 45 were, Pearpolo-5/Jyoti (88.30).L-116/Jyoti (80.89), Pearpolo-5/L116(72.83), Jyoti/PB-01(45.35), AP-3/VRPE24(39.80),VRPE-4 /Sonali-10(36.15), and the better parent heterosis as estimated for days to maturity was significant and negative for all the hybrids. The range of variation from -0.88 to -7.54exhibiting desirable heterosis; out of these range 33 hybrids were desirable and significant. Some of these were Rachana/Jyoti (-7.54), VRPE-24/PB-01(-6.60), VRPE32/PB-01, (-6.45), Rachana/PB-01, (-6.24). The heterosis over better parent for pod length were positively associated and ranged from 1.00 to 22.40 . The highest value of heterosis were observed in AP-3/Rachana (22.42), KSP-2/Rachana (20.26), L-116/KSP2(16.94), AP-3/KSP-2(16.70), Pearpolo5/AP-3 (6.56) from the Table 8. It is clearly shown that the heterosis over better parent for Seeds per pod were ranged from -1.44 to 21.11 per cent exhibiting desirable and significant hybrid in eight cases. Heterosis over better parent for number of pod density ranged from-2.16 to 44.56 percent showing desirable and significant hybrids in fourteen cases. Out of these, high heterosis was observed in AP-3/KSP-2(44.56), Pearpol5/AP-3(34.76), AP-3 /Rachana (34.44), L116/KSP-2(33.98), VRPE-32/L-116(28.25), VRPE-32/L-116 (28.25), Jyoti/KSP-2(26.21), Crosses. Heterosis over better parent for $100-$ seed weight ranged from -0.48to 18.94 percent observed desirable and significant in twenty three cases.100-seed weight was positively associated so highly significant and positively associated hybrids were VRPE32/VRPE-24 (18.94), Pearpolo-5/AP-3 (17.09), Pearpolo-5/PB-01 (12.84), AP3/Jyoti (11.79), VRPE-24/PB-01(11.76) and VRPE-24/Jyoti (10.36). Similar results were also reported by Alam et al., (2008). Pods per plant exhibited the heterosis ranged from 0.08 to 26.90 per cent. Significant and positive heterosis for Jyoti/Sonali-10(26.90), VRPE-24/PB-01(26.85), L-116/AP-3(25.910, AP 3/Jyoti (25.06), VRPE-32/AP-3(18.33). Heterosis over better parent for yield per plant ranged from -2.56 to 4.40 per cent. Similar results were reported by Mehmet et al., (2008) for yield per plant. Likewise, similar finding had also reported by Ceyhan et al., (2003) in pea and Motamedi et al., (2014). Significant and positive heterosis for Pearpolo-5/Sonali-10(4.40), AP-3/Sonali-10 (4.49), VRPE-24/PB-01(2.56), were most considerable.

\section{Means and variability in parents, $F_{1} S$}

The mean values and range for all the traits in parents their $\mathrm{F}_{1} \mathrm{~S}$ hybrids generation mentioned in Table 2. The mean performance of $F_{1}$ hybrids was higher than parents for number of primary branches /plant, pod length, pod per plant, pod density. Revealed maximum variation within parents for days to $75 \%$ flowering, plant height, days to maturity, but their magnitude varied from character to character. The variability among parents was fairly high for plant height (62.21-82.76), days to maturity (92-104), yield /plant (70.3982.12) followed by pod per plant (50.1258.97). The variation in $\mathrm{F}_{1}$ progenies was maximum for plant height (60.40-75.87), followed by days to maturity (91.33-103.66), yield per plant (62.01-79.37), 100 -seed weight (11.43-15.77) pod density (0.82-1.26) primary branches per plant (2.46-5.30) Pod per plant (53.36-71.82) seed per pod (6.639.53) Pod length (6.83-9.34).

\section{Combining ability analysis}

Analysis of variance for combining ability: The analysis of variance for combining ability was carried out for all the characters in $F_{1}$ generations and result are presented in Table 
1. Griffings (1956b) presented a model to show that variance for gca involves additive and additive $\mathrm{x}$ additive gene action. The mean squares due to general combining ability (GCA) effects and specific combining ability (SCA) effect were found highly numbers for all the characters in $F_{1}$ generations. The magnitude of estimates of GCA variances were higher for days to $75 \%$ flowering, plant height, primary branches per plant, days to maturity, seeds per pod, pod density, pod per plant and yield per plant than the respective estimates of SCA variances $F_{1}$ in generations. General predictability ratio did not reach near to unity for any of the traits in $F_{1}$ generations.

In the previous studies Singh et al., (2010), Motamedi et al., (2014) and Singh et al., (2014) revealed that estimates of SCA variances were higher than GCA variances for all the traits studied, thus indicating predominance of non-additive gene action for these traits

Table.1 ANOVA for combining ability and related statistics of 10 characters in a 10 parent HalfDiallel cross in $\mathrm{F}_{1}$ generations of pea

\begin{tabular}{|c|c|c|c|c|c|c|}
\hline \multirow[t]{2}{*}{$\begin{array}{l}\text { Source of } \\
\text { variation }\end{array}$} & d.f. & $\begin{array}{l}\text { Days to } 75 \% \\
\text { flowering }\end{array}$ & $\begin{array}{l}\text { Plant } \\
\text { Height }(\mathrm{cm})\end{array}$ & $\begin{array}{l}\text { Primary } \\
\text { Branches/ } \\
\text { Plant }\end{array}$ & $\begin{array}{l}\text { Pod } \\
\text { Length } \\
(\mathrm{cm})\end{array}$ & Seeds/ Pod \\
\hline & & $\mathrm{F}_{1}$ & $\mathrm{~F}_{1}$ & $F_{1}$ & $\mathrm{~F}_{1}$ & $\mathrm{~F}_{1}$ \\
\hline GCA & 9 & 4.14 & 10.26 & 1.07 & 0.71 & 0.94 \\
\hline SCA & 45 & 11.83 & 23.97 & 0.53 & 0.37 & 0.61 \\
\hline Error & 108 & 0.92 & 0.02 & 0.00 & 0.00 & 0.01 \\
\hline$\sigma_{g}^{2}$ & & -0.64 & -1.14 & 0.04 & 0.03 & 0.02 \\
\hline$\sigma_{s}^{2}$ & & 10.90 & 23.95 & 0.52 & 0.36 & 0.59 \\
\hline GPR & & -0.13 & -010 & 0.14 & 0.14 & 0.08 \\
\hline
\end{tabular}

Table.1 Contd.

\begin{tabular}{|c|c|c|c|c|c|c|}
\hline \multirow[t]{2}{*}{$\begin{array}{l}\text { Source of } \\
\text { variation }\end{array}$} & \multirow[t]{2}{*}{ d.f. } & Pod Density $(\mathrm{cm})$ & Pods/ Plant & $\begin{array}{l}\text { Days to } \\
\text { Maturity }\end{array}$ & $\begin{array}{l}100 \text { seed } \\
\text { Weight }(\mathrm{g})\end{array}$ & $\begin{array}{l}\text { Yield/ Plant } \\
\text { G(kg) }\end{array}$ \\
\hline & & $\mathrm{F}_{1}$ & $\mathrm{~F}_{1}$ & $\mathrm{~F}_{1}$ & $\mathrm{~F}_{1}$ & $\mathrm{~F}_{1}$ \\
\hline GCA & 9 & 0.01 & 56.65 & 13.36 & 1.59 & 21.42 \\
\hline SCA & 45 & 0.02 & 26.67 & 8.94 & 1.51 & 22.26 \\
\hline Error & 108 & 0.00 & 0.24 & 0.26 & 0.03 & 0.08 \\
\hline$\sigma_{g}^{2}$ & & -0.00 & 2.49 & 0.36 & 0.00 & -0.06 \\
\hline$\sigma_{s}^{2}$ & & 0.02 & 26.43 & 8.67 & 1.47 & 22.18 \\
\hline GPR & & -0.03 & 0.15 & 0.07 & 0.00 & -0.00 \\
\hline
\end{tabular}

* Significant at $5 \%$ level; ** significant at $1 \%$ level; GCA= general combining ability; SCA= specific combining ability; $\boldsymbol{\sigma}_{\mathrm{g}}{ }^{2}=$ estimates of gca variance; $\boldsymbol{\sigma}_{\mathrm{s}}{ }_{\mathrm{s}}=$ estimates of sca variance; GPR =general predictability ratio 
Table.2 Estimates of GCA effects corresponding mean performance of the parents for 10 characters in a 10 parents Half-Diallel cross in $\mathrm{F}_{1}$ generations of pea

\begin{tabular}{|c|c|c|c|c|c|c|c|c|c|c|}
\hline \multirow[t]{3}{*}{ Parents } & \multicolumn{2}{|c|}{ Day to $75 \%$ Flowering } & \multicolumn{2}{|c|}{ Plant Height (cm) } & \multicolumn{2}{|c|}{$\begin{array}{c}\text { Primary Branches/ } \\
\text { Plant }\end{array}$} & \multicolumn{2}{|c|}{ Pod Length (cm) } & \multicolumn{2}{|c|}{ Seeds/ Pod } \\
\hline & GCA effect & Mean & $\begin{array}{l}\text { GCA } \\
\text { effect }\end{array}$ & Mean & $\begin{array}{l}\text { GCA } \\
\text { effect }\end{array}$ & Mean & $\begin{array}{l}\text { GCA } \\
\text { effect }\end{array}$ & Mean & $\begin{array}{l}\text { GCA } \\
\text { effect }\end{array}$ & Mean \\
\hline & $\mathrm{F}_{1}$ & & $F_{1}$ & & $F_{1}$ & & $F_{1}$ & & $F_{1}$ & \\
\hline Pear Polo-5 & $-1.08 * * *$ & 72.33 & $-0.51 * * *$ & 71.46 & $0.26 * * *$ & 3.30 & $0.37 * * *$ & 8.52 & $0.15^{* * * *}$ & 7.66 \\
\hline VRPE-32 & -0.31 & 72.66 & $-1.07 * * *$ & 68.82 & $0.25^{* * * *}$ & 4.30 & $0.13^{* * *}$ & 7.74 & $0.09 * *$ & 8.53 \\
\hline L-116 & $0.63^{*}$ & 84.33 & $-1.23 * * *$ & 71.84 & $0.15^{* * * *}$ & 2.83 & $0.26^{* * *}$ & 8.81 & $-0.23 * * *$ & 7.36 \\
\hline AP-3 & 0.41 & 79.66 & $0.23 * * *$ & 74.81 & -0.01 & 3.46 & $-0.24 * * *$ & 6.74 & $-0.20 * * *$ & 8.73 \\
\hline Rachana & $-0.61 *$ & 74.66 & $1.04 * * *$ & 82.76 & $-0.48 * * *$ & 3.26 & $-0.15 * * *$ & 7.23 & $0.35^{* * * *}$ & 8.60 \\
\hline VRPE-24 & 0.43 & 80.33 & $0.58 * * *$ & 74.79 & $-0.11 * * *$ & 3.06 & $0.07 * *$ & 9.26 & 0.13 *** & 8.43 \\
\hline Jyoti & 0.35 & 80.00 & $-0.98 * * *$ & 62.21 & $-0.53 * * *$ & 2.40 & $-0.22 * * *$ & 7.68 & $-0.25 * * *$ & 8.50 \\
\hline PB-01 & -0.50 & 75.00 & $0.52 * * *$ & 81.57 & $0.13^{* * * *}$ & 3.33 & $0.12 * * *$ & 8.86 & $0.41 * * *$ & 8.50 \\
\hline KSP-2 & 0.16 & 76.33 & 0.01 & 74.50 & $0.31 * * *$ & 4.53 & $-0.42 * * *$ & 6.77 & $-0.44 * * *$ & 7.90 \\
\hline Sonali-10 & 0.52 & 80.33 & $1.41 * * *$ & 82.27 & 0.02 & 4.03 & $0.08^{* *}$ & 9.37 & -0.02 & 6.70 \\
\hline Gi--Gj at $95 \%$ & $0.89 * * *$ & & $0.15 * * *$ & & $0.05^{* * * *}$ & & $0.08 * * *$ & & $0.11 * * *$ & \\
\hline Gi--Gj at $99 \%$ & $1.27 * * *$ & & $0.22 * * *$ & & $0.08 * * *$ & & $0.12 * * *$ & & $0.17 * * *$ & \\
\hline
\end{tabular}

Table. 2 Contd.

\begin{tabular}{|c|c|c|c|c|c|c|c|c|c|c|}
\hline \multirow[t]{3}{*}{ Parents } & \multicolumn{2}{|c|}{ Pod Density (cm) } & \multicolumn{2}{|c|}{ Pods/ Plant } & \multicolumn{2}{|c|}{ Days to Maturity } & \multicolumn{2}{|c|}{ Yield/ Plant G(kg) } & \multicolumn{2}{|c|}{100 seed Weight (g) } \\
\hline & GCA effect & Mean & GCA effect & Mean & GCA effect & Mean & GCA effect & Mean & $\begin{array}{l}\text { GCA } \\
\text { effect }\end{array}$ & Mean \\
\hline & $\mathrm{F}_{1}$ & & $\mathrm{~F}_{1}$ & & $\mathrm{~F}_{1}$ & & $\mathrm{~F}_{1}$ & & $\mathrm{~F}_{1}$ & \\
\hline Pear Polo-5 & $0.02 * *$ & 1.10 & $-3.06 * * *$ & 52.49 & $-0.93 * * *$ & 95.00 & $-0.31 * * *$ & 71.28 & $-0.32 * * *$ & 12.70 \\
\hline VRPE-32 & 0.00 & 0.90 & $-1.60 * * *$ & 56.45 & $-1.05 * * *$ & 97.66 & $-0.20^{*}$ & 75.82 & -0.04 & 13.02 \\
\hline L-116 & $0.06^{* * * *}$ & 1.19 & $-1.33 * * *$ & 50.12 & $0.58 * * *$ & 101.66 & $-1.63 * * *$ & 70.39 & -0.05 & 14.89 \\
\hline AP-3 & -0.01 & 0.76 & $-2.36^{* * *}$ & 47.75 & $-0.55 * * *$ & 94.66 & $0.30^{* * * *}$ & 77.09 & $-0.36^{* * *}$ & 11.96 \\
\hline Rachana & $-0.06^{* * * *}$ & 0.84 & $-1.30^{* * *}$ & 54.65 & $2.31^{* * *}$ & 104.33 & $-1.78 * * *$ & 78.48 & $0.36^{* * *}$ & 15.74 \\
\hline VRPE-24 & $-0.02 * *$ & 1.09 & $1.32 * * *$ & 56.77 & $-0.66 * * *$ & 98.00 & $-0.84 * * *$ & 72.67 & -0.01 & 12.67 \\
\hline Jyoti & $0.01^{*}$ & 0.90 & $2.90 * * *$ & 54.33 & $0.33^{*}$ & 103.33 & $0.27 * * *$ & 80.64 & 0.05 & 13.86 \\
\hline PB-01 & $-0.04 * * *$ & 1.04 & $2.02^{* * * *}$ & 56.36 & 0.89 & 104.00 & $2.87 * * *$ & 82.12 & $-0.46^{* * *}$ & 11.73 \\
\hline KSP-2 & 0.00 & 0.85 & $2.51 * * *$ & 58.97 & -0.10 & 97.66 & $0.44 * * *$ & 77.93 & 0.09 & 14.75 \\
\hline Sonali-10 & 0.02 & 1.40 & $0.89 * * *$ & 57.19 & $-0.82 * * *$ & 92.00 & $0.87 * * *$ & 73.90 & $0.77^{* * *}$ & 15.69 \\
\hline Gi--Gj at $95 \%$ & $0.02 * * *$ & & $0.45^{* * * *}$ & & $0.47 * * *$ & & $0.26^{* * *}$ & & $0.18^{* * * *}$ & \\
\hline Gi--Gj at $99 \%$ & $0.03 * * *$ & & $0.65^{* * * *}$ & & $0.68 * * *$ & & $0.38 * * *$ & & $0.26^{* * *}$ & \\
\hline
\end{tabular}

Significant at $5 \%$ level; $* *$ significant at $1 \%$ level

Table.3 Best general combiners on the basis of per se performance and GCA effect for 10 characters in a 10 parent Half-Diallel cross in pea

\begin{tabular}{|c|c|c|c|}
\hline \multirow[t]{2}{*}{ Characters } & \multirow[b]{2}{*}{ Per se performance } & Gca & \multirow[b]{2}{*}{ Common parent } \\
\hline & & $\mathrm{F}_{1}$ & \\
\hline Day to $75 \%$ Flowering & Pearpolo-5, VRPE-32, Rachana, PB-01, KSP-2 & $\begin{array}{l}\text { Pearpolo-5, VRPE-32, Rachana, PB- } \\
01\end{array}$ & $\begin{array}{l}\text { Pearpolo-5, VRPE-32, } \\
\text { Rachana, PB-01 }\end{array}$ \\
\hline Plant Height $(\mathrm{cm})$ & Pearpolo-5, VRPE-32, L-116, Jyoti, KSP-2 & L-116, VRPE-32, Pearpolo-5, Jyoti & $\begin{array}{l}\text {-116, VRPE-32, Pearpolo-5, } \\
\text { Jyoti }\end{array}$ \\
\hline Primary Branches/ Plant & KSP-2, VRPE-32, Sonali-10, AP-3, PB-01 & KSP-2, Pearpolo-5, VRPE-32, PB-01 & KSP-2, VRPE-32, PB-01 \\
\hline Pod Length $(\mathrm{cm})$ & Sonali-10, VRPE-32, PB-01, L-116, Pearpolo-5, & $\begin{array}{l}\text { Pearpolo-5, L-116, VRPE-32, PB-01, } \\
\text { Sonali-5 }\end{array}$ & $\begin{array}{l}\text { Pearpolo-5, L-116, VRPE-32, } \\
\text { PB-01, }\end{array}$ \\
\hline Seeds/ Pod & AP-3, Rachana, VRPE-32, Jyoti, PB-01 & $\begin{array}{l}\text { PB-01, Rachana, Pearpolo-5, VRPE- } \\
32\end{array}$ & PB-01, Rachana \\
\hline Pod Density $(\mathrm{cm})$ & Sonali-10, Pearpolo-5, L-116, VRPE-32, PB-01, & L-116, Sonali-10, Peearpolo-5, Jyoti & L-116, Sonali-10, Peearpolo-5, \\
\hline Pods/ Plant & KSP-2, Sonali-10, VRPE-32, VRPE-24, PB-01 & Jyoti, KSP-2, PB-01, VRPE-24 & KSP-2, PB-01, VRPE-24 \\
\hline Days to Maturity & Sonali-10, AP-3, Pearpolo-5, KSP-2 & $\begin{array}{l}\text { VRPE32, Pearpolo-5, Sonali-10, } \\
\text { VRPE-24, KSP-2 }\end{array}$ & $\begin{array}{l}\text { VRPE32, Pearpolo-5, Sonali- } \\
\text { 10, VRPE-24, }\end{array}$ \\
\hline Yield/ Plant G(kg) & PB-01, Jyoti, Rachana, AP-3, KSP-2 & PB-01, KSP-2, Sonali-10, AP-3 & PB-01, KSP-2, AP-3 \\
\hline 100 seed Weight $(\mathrm{g})$ & Rachana, Sonali-10, L-116, KSP-2, Jyoti & Rachana, Sonali-10, KSP-2, Jyoti & Sonali-10, KSP-2, Jyoti \\
\hline
\end{tabular}


Table.4 Estimation of SCA and corresponding mean performance for 10 characters in a 10 parents half diallel cross in $\mathrm{F}_{1}$ generations of pea

\begin{tabular}{|c|c|c|c|c|c|c|c|c|c|c|}
\hline \multirow[t]{2}{*}{ Cross combination } & \multicolumn{2}{|c|}{$\begin{array}{l}\text { Days to } 75 \% \\
\text { Flowering }\end{array}$} & \multicolumn{2}{|c|}{ Plant height $(\mathrm{cm})$} & \multicolumn{2}{|c|}{$\begin{array}{l}\text { Primary } \\
\text { branches/plant }\end{array}$} & \multicolumn{2}{|l|}{$\begin{array}{l}\text { Pod length } \\
(\mathrm{cm})\end{array}$} & \multicolumn{2}{|l|}{ Seeds/pod } \\
\hline & SCA & Mean & SCA & Mean & SCA & Mean & SCA & Mean & SCA & Mean \\
\hline Pear Polo-5*VRPE-32 & -0.39 & 70.33 & $-6.83^{* *}$ & 61.23 & $0.12^{*}$ & 4.60 & $0.43 * * *$ & 9.12 & 0.18 & 8.43 \\
\hline Pear Polo-5*L-116 & -0.67 & 71.00 & $-7.49 * *$ & 60.41 & $0.92 * * *$ & 5.30 & 0.10 & 8.92 & $0.48 * * *$ & 8.40 \\
\hline Pear Polo-5*AP-3 & $1.88^{*}$ & 73.33 & $-0.58 * *$ & 68.79 & 0.05 & 4.27 & $1.03 * * *$ & 9.34 & $-0.54 * * *$ & 7.40 \\
\hline Pear Polo-5*RACHANA & 0.58 & 71.00 & $-0.84 * *$ & 69.34 & $-0.33 * * *$ & 3.40 & $-0.28 * *$ & 8.12 & $0.96 * * *$ & 9.47 \\
\hline Pear Polo-5*VRPE-24 & -2.14 & 69.33 & $0.47 * *$ & 70.19 & 0.02 & 4.13 & $-0.75^{* * *}$ & 7.87 & $1.24 * * *$ & 9.53 \\
\hline Pear Polo-5*JYOTI & $-2.72 * *$ & 68.67 & $3.52 * *$ & 71.68 & $1.67 * * *$ & 5.37 & 0.12 & 8.46 & $-0.86^{* * *}$ & 7.03 \\
\hline Pear Polo-5*PB-01 & -0.86 & 69.67 & $6.20 * *$ & 75.87 & -0.02 & 4.33 & $0.45^{* * *}$ & 9.13 & $-0.38 * *$ & 8.20 \\
\hline Pear Polo-5*KSP-2 & -0.86 & 70.33 & $1.51 * *$ & 70.66 & $0.29 * * *$ & 4.83 & $-0.36 * * *$ & 7.77 & $-0.37 * *$ & 7.33 \\
\hline Pear Polo-5*sonali-10 & 0.43 & 72.00 & $-1.63 * *$ & 68.88 & $-0.34 * * *$ & 3.90 & 0.03 & 8.67 & $0.56^{* * *}$ & 8.70 \\
\hline VRPE-32*L-116 & -0.44 & 72.00 & $6.40^{* *}$ & 73.74 & $0.16^{* *}$ & 4.53 & $0.44 * * *$ & 9.02 & $-1.16^{* * * *}$ & 6.70 \\
\hline VRPE-32*AP-3 & 0.43 & 72.67 & $2.52 * *$ & 71.34 & $0.53 * * *$ & 4.73 & $0.19 *$ & 8.25 & $0.50 * * *$ & 8.40 \\
\hline VRPE-32*RACHANA & 0.46 & 71.67 & $-3.75 * *$ & 65.87 & -0.09 & 3.63 & $0.42 * * *$ & 8.58 & $-0.91 * * *$ & 7.53 \\
\hline VRPE-32*VRPE-24 & 0.41 & 72.67 & $-0.69 * *$ & 68.47 & $0.43^{* * *}$ & 4.53 & $0.21 *$ & 8.60 & 0.13 & 8.37 \\
\hline VRPE-32*JYOTI & 0.16 & 72.33 & $2.52 * *$ & 70.12 & $-0.41 * * *$ & 3.27 & $-0.72 * * *$ & 7.37 & $0.09 *$ & 7.93 \\
\hline VRPE-32*PB-01 & 1.02 & 72.33 & $-4.47 * *$ & 64.63 & $0.48^{* * *}$ & 4.83 & $0.46^{* * *}$ & 8.89 & 0.28 & 8.80 \\
\hline VRPE-32*KSP-2 & -0.64 & 71.33 & $1.28 * *$ & 69.88 & -0.09 & 4.43 & -0.03 & 7.85 & $-0.19^{* *}$ & 7.47 \\
\hline VRPE-32*sonali-10 & $-3.33 * *$ & 69.00 & $0.35 * *$ & 70.34 & $-0.80 * * *$ & 3.43 & -0.01 & 8.38 & $0.39 * * *$ & 8.47 \\
\hline L-116*AP-3 & $-3.17 * *$ & 70.00 & $-1.20 * *$ & 67.45 & $0.99 * * *$ & 5.10 & $-0.72 * * *$ & 7.47 & $-0.92 * * *$ & 6.63 \\
\hline L-116*RACHANA & $-3.14 * *$ & 69.00 & $-6.22 * *$ & 63.24 & $-0.76 * * *$ & 2.87 & 0.16 & 8.46 & $1.01 * * *$ & 9.13 \\
\hline L-116*VRPE-24 & $-3.86 * * *$ & 69.33 & $2.43 * *$ & 71.44 & $-0.23 * * *$ & 3.77 & $-0.39 * * *$ & 8.12 & 0.47 & 8.37 \\
\hline L-116*JYOTI & $-4.44 * *$ & 68.67 & $2.86 * *$ & 70.29 & $1.15^{* * *}$ & 4.73 & $-0.59 * * *$ & 7.63 & 0.06 & 7.53 \\
\hline L-116*PB-01 & $-1.92 *$ & 70.33 & $0.45^{* *}$ & 69.39 & $-0.41 * * *$ & 3.83 & $0.36^{* * * *}$ & 8.93 & 0.12 & 8.30 \\
\hline L-116*KSP-2 & -0.25 & 72.67 & $-4.57 * *$ & 63.86 & $-0.16^{* *}$ & 4.27 & $1.08 * * *$ & 9.11 & $-0.68 * * *$ & 6.63 \\
\hline L-116*sonali-10 & $-3.94 * *$ & 69.33 & $-1.99 * *$ & 67.83 & $1.19 * * *$ & 5.33 & $-0.67 * * *$ & 7.85 & $0.96^{* * * *}$ & 8.70 \\
\hline AP-3*RACHANA & -0.58 & 71.33 & $1.70 * *$ & 72.64 & $-0.66 * * *$ & 2.80 & $0.77 * * *$ & 8.55 & -0.14 & 8.00 \\
\hline AP-3*VRPE-24 & $-3.97 * *$ & 69.00 & $-4.65 * *$ & 65.83 & $0.73 * * *$ & 4.57 & -0.09 & 7.92 & $-1.32 * * *$ & 6.60 \\
\hline AP-3*JYOTI & $-2.56 * *$ & 70.33 & -0.02 & 68.88 & $-0.24 * * *$ & 3.17 & $0.61 * * *$ & 8.33 & 0.12 & 7.67 \\
\hline AP-3*PB-01 & -1.69 & 70.33 & $-6.67 * *$ & 63.74 & $0.52 * * *$ & 4.60 & $-0.40 * * *$ & 7.65 & $0.85 * * *$ & 9.07 \\
\hline $\mathrm{AP}-3 * \mathrm{KSP}-2$ & -1.03 & 71.67 & $-0.53 * *$ & 69.37 & $0.50 * * *$ & 4.77 & $0.37 * * *$ & 7.88 & $-0.65 * * *$ & 6.70 \\
\hline AP-3*sonali-10 & $-2.72 * *$ & 70.33 & 0.07 & 71.37 & $-1.50 * * *$ & 2.47 & 0.11 & 8.12 & -0.17 & 7.60 \\
\hline RACHANA*VRPE-24 & -0.61 & 71.33 & $-5.73 * *$ & 65.56 & $0.33^{* * *}$ & 3.70 & $0.22^{*}$ & 8.32 & -0.11 & 8.37 \\
\hline RACHANA*JYOTI & -0.53 & 71.33 & -0.26 & 69.45 & $-0.24 * * *$ & 2.70 & $-0.24 * *$ & 7.56 & $-1.33 * * *$ & 6.77 \\
\hline RACHANA*PB-01 & -0.67 & 70.33 & $-1.56 * *$ & 69.67 & $0.26 * * *$ & 3.87 & $-0.24 * *$ & 7.90 & -0.23 & 8.53 \\
\hline RACHANA*KSP-2 & -1.67 & 70.00 & $1.92 * *$ & 72.64 & $0.91^{* * *}$ & 4.70 & $0.81 * * *$ & 8.42 & $0.58 * * *$ & 8.50 \\
\hline RACHANA*sonali-10 & -1.36 & 70.67 & $-7.27 * *$ & 64.84 & 0.03 & 3.53 & $-0.37 * * *$ & 7.73 & $0.40 * *$ & 8.73 \\
\hline VRPE-24*JYOTI & -1.25 & 71.67 & $0.99 * *$ & 70.25 & $-0.48 * * *$ & 2.83 & $0.39 * * *$ & 8.43 & $-1.21 * * *$ & 6.67 \\
\hline VRPE-24*PB-01 & -0.39 & 71.67 & -0.15 & 70.62 & $0.25 * * *$ & 4.23 & $-1.40 * * *$ & 6.97 & $-0.27 *$ & 8.27 \\
\hline VRPE-24*KSP-2 & -1.39 & 71.33 & $1.49 * *$ & 71.75 & $-0.69 * * *$ & 3.47 & -0.18 & 7.67 & $1.34 * * *$ & 9.03 \\
\hline VRPE-24*sonali-10 & -1.42 & 71.67 & $-2.11 * *$ & 69.53 & $0.96 * * *$ & 4.83 & 0.10 & 8.44 & $-0.61 * * *$ & 7.50 \\
\hline JYOTI*PB-01 & $-1.97 *$ & 70.00 & $-2.61 * *$ & 66.57 & $0.60 * * *$ & 4.17 & 0.09 & 8.18 & $0.90 * * *$ & 9.07 \\
\hline JYOTI*KSP-2 & 0.35 & 73.00 & $0.33^{*}$ & 69.02 & $-0.44 * * *$ & 3.30 & 0.04 & 7.58 & $-0.50 * * *$ & 6.80 \\
\hline JYOTI*sonali-10 & -1.33 & 71.67 & $3.58 * * *$ & 73.66 & $-0.61 * * *$ & 2.83 & $0.38 * * *$ & 8.43 & $0.74 * * *$ & 8.47 \\
\hline PB-01*KSP-2 & -1.44 & 70.33 & $-6.71 * *$ & 63.48 & $-0.57 * * *$ & 3.83 & $0.41 * * *$ & 8.30 & $-0.97 * * *$ & 7.00 \\
\hline PB-01*sonali-10 & 0.18 & 72.33 & $-6.20 * *$ & 65.39 & $0.65 * * *$ & 4.77 & $-0.62 * * *$ & 7.76 & $0.34 * *$ & 8.73 \\
\hline KSP-2*sonali-10 & -0.81 & 72.00 & $-4.37 * *$ & 66.71 & $0.36^{* * * *}$ & 4.67 & $-1.02 * * *$ & 6.82 & -0.10 & 7.43 \\
\hline
\end{tabular}

Significant at $5 \%$ level; ** significant at $1 \%$ level 
Int.J.Curr.Microbiol.App.Sci (2017) 6(12): 1574-1585

Table.4 Contd.

\begin{tabular}{|c|c|c|c|c|c|c|c|c|c|c|}
\hline \multirow[t]{2}{*}{ Cross combination } & \multicolumn{2}{|c|}{ Pod Density $(\mathrm{cm})$} & \multicolumn{2}{|c|}{ Pods/plant } & \multicolumn{2}{|c|}{ Days to maturity } & \multicolumn{2}{|c|}{ Yield/ Plant G(kg) } & \multicolumn{2}{|c|}{100 -seed weight $(\mathrm{g})$} \\
\hline & SCA & Mean & SCA & Mean & SCA & Mean & SCA & Mean & SCA & Mean \\
\hline Pear Polo-5*VRPE-32 & 0.02 & 1.08 & -0.61 & 55.41 & $-2.64 * * *$ & 92.67 & $-4.22 * * *$ & 66.57 & $-0.48 *$ & 12.85 \\
\hline Pear Polo-5*L-116 & $-0.06^{*}$ & 1.06 & 0.35 & 56.66 & $-1.28 *$ & 95.67 & $1.29 * * *$ & 70.66 & $0.54 * *$ & 13.86 \\
\hline Pear Polo-5*AP-3 & $0.21^{* * *}$ & 1.26 & $2.20^{* * *}$ & 57.48 & $-1.80 * * *$ & 94.00 & $1.57 * * *$ & 72.87 & $1.42 * * *$ & 14.44 \\
\hline Pear Polo-5*RACHANA & $-0.13 * * *$ & 0.85 & $1.01 *$ & 57.35 & $-3.33 * * *$ & 95.33 & $-1.16^{* * *}$ & 68.05 & 0.12 & 13.86 \\
\hline Pear Polo-5*VRPE-24 & $-0.21 * * *$ & 0.82 & $1.01 *$ & 59.98 & $-2.69 * * *$ & 93.00 & $-0.65 *$ & 69.49 & 0.28 & 13.64 \\
\hline Pear Polo-5*PB-01 & $0.09^{* * *}$ & 1.11 & $-1.05^{*}$ & 58.62 & -0.25 & 97.00 & $0.58^{*}$ & 74.45 & $0.87 * * *$ & 13.79 \\
\hline Pear Polo-5*KSP-2 & -0.00 & 1.05 & -0.18 & 59.97 & $5.08 * * *$ & 101.33 & $-4.84 * * *$ & 66.60 & 0.24 & 13.72 \\
\hline Pear Polo-5*sonali-10 & $-0.09 * *$ & 0.99 & $1.24 * *$ & 59.78 & $6.47 * * *$ & 102.00 & $3.91 * * *$ & 75.78 & $-1.50 * * *$ & 12.64 \\
\hline VRPE-32*L-116 & $0.24 * * *$ & 1.35 & $2.91 * * *$ & 60.68 & $-2.50 * * *$ & 94.33 & $-0.68 *$ & 68.78 & $-1.96^{* * * *}$ & 11.63 \\
\hline VRPE-32*AP-3 & -0.02 & 1.00 & $4.92 * * *$ & 61.65 & $0.97 *$ & 96.67 & $-3.83^{* * *}$ & 67.57 & $1.32 * * *$ & 14.62 \\
\hline VRPE-32*PB-01 & 0.02 & 1.01 & $-5.46^{* * *}$ & 55.66 & $-2.80 * * *$ & 94.33 & $-2.78 * * *$ & 71.19 & 0.23 & 13.42 \\
\hline VRPE-32*KSP-2 & 0.01 & 1.05 & $-3.85 * * *$ & 57.76 & 0.52 & 96.67 & $1.82 * * *$ & 73.38 & $-1.06 * * *$ & 12.68 \\
\hline VRPE-32*sonali-10 & $-0.07 * *$ & 0.98 & 0.72 & 60.71 & $-4.08 * * *$ & 91.33 & $2.62 * * *$ & 74.60 & $1.12 * * *$ & 15.55 \\
\hline L-116*AP-3 & 0.038 & 1.12 & $4.61 * * *$ & 61.62 & $-2.00 * * *$ & 95.33 & $-4.60 * * *$ & 65.38 & $1.37 * * *$ & 14.65 \\
\hline L-116*RACHANA & $-0.11 * * *$ & 0.92 & $5.91 * * *$ & 63.99 & $3.47 * * *$ & 103.67 & -0.40 & 67.48 & $0.70^{* * * *}$ & 14.71 \\
\hline L-116*VRPE-24 & $-0.11 * * *$ & 0.96 & $3.97 * * *$ & 64.68 & 0.10 & 97.33 & $2.47 * * *$ & 71.30 & $-1.96 * * *$ & 11.66 \\
\hline L-116*JYOTI & $-0.10^{* * *}$ & 1.01 & $3.28^{* * *}$ & 65.56 & $-2.55^{* * *}$ & 95.67 & $-1.45^{* * *}$ & 68.49 & -0.04 & 13.65 \\
\hline L-116*PB-01 & 0.015 & 1.07 & $-2.98 * * *$ & 58.41 & 0.22 & 99.00 & $2.15^{* * *}$ & 74.70 & $-0.55^{* *}$ & 12.63 \\
\hline L-116*KSP-2 & $0.27 * * *$ & 1.37 & $-2.02 * * *$ & 59.86 & -0.78 & 97.00 & $1.25^{* * *}$ & 71.38 & $-2.05^{* * *}$ & 11.68 \\
\hline L-116*sonali-10 & $-0.22 * * *$ & 0.90 & -0.21 & 60.05 & $-1.05^{*}$ & 96.00 & $-4.74 * * *$ & 65.80 & $1.35 * * *$ & 15.77 \\
\hline RACHANA*JYOTI & $0.13 * * *$ & 1.11 & -0.86 & 61.45 & $-3.94 * * *$ & 96.00 & $-7.78 * * *$ & 62.01 & $-2.58 * * *$ & 11.54 \\
\hline RACHANA*PB-01 & 0.00 & 0.92 & $4.17 * * *$ & 65.60 & $-2.83 * * *$ & 97.67 & $-6.70 * * *$ & 65.69 & $1.65 * * *$ & 15.25 \\
\hline RACHANA*KSP-2 & 0.01 & 0.99 & $-3.10 * * *$ & 58.82 & $-3.50 * * *$ & 96.00 & $-4.85^{* * *}$ & 65.12 & 0.30 & 14.46 \\
\hline RACHANA*sonali-10 & $-0.11 * * *$ & 0.88 & $-1.88 * * *$ & 58.41 & $-2.78 * * *$ & 96.00 & $-0.92 * *$ & 69.47 & $-2.51 * * *$ & 12.32 \\
\hline VRPE-24*JYOTI & $0.23 * * *$ & 1.26 & $4.59 * * *$ & 69.53 & $-0.97 *$ & 96.00 & $5.09 * * *$ & 75.83 & $0.90 * * *$ & 14.64 \\
\hline VRPE-24*PB-01 & $-0.13 * * *$ & 0.84 & 7.69 *** & 71.76 & $-3.19 * * *$ & 94.33 & $6.04 * * *$ & 79.38 & $0.41 *$ & 13.64 \\
\hline VRPE-24*KSP-2 & $-0.16^{* * *}$ & 0.85 & $6.06 * * *$ & 70.61 & $-1.86^{* * *}$ & 94.67 & $-5.14 * * *$ & 65.76 & $0.44 *$ & 14.23 \\
\hline VRPE-24*sonali-10 & $0.08 * *$ & 1.12 & $-6.02 * * *$ & 56.90 & $1.19 *$ & 97.00 & $-6.99 * * *$ & 64.34 & $0.79 * * *$ & 15.25 \\
\hline JYOTI*PB-01 & $-0.11 * * *$ & 0.89 & $6.18 * * *$ & 71.82 & $1.47 * *$ & 100.00 & $-2.44 * * *$ & 72.01 & $0.40 *$ & 13.70 \\
\hline JYOTI*KSP-2 & $0.05^{*}$ & 1.11 & $2.63 * * *$ & 68.76 & $-1.53 * *$ & 96.00 & $-4.30 * * *$ & 67.72 & 0.12 & 13.98 \\
\hline JYOTI*sonali-10 & $0.07 *$ & 1.15 & $6.26^{* * * *}$ & 70.77 & $-1.14^{*}$ & 95.67 & $-1.80 * * *$ & 70.65 & 0.15 & 14.69 \\
\hline PB-01*KSP-2 & $0.18^{* * *}$ & 1.18 & $6.56^{* * *}$ & 71.81 & $-3.08 * *$ & 95.00 & $-0.87 * *$ & 73.75 & $0.75^{* * *}$ & 14.09 \\
\hline PB-01*sonali-10 & $-0.13 * * *$ & 0.88 & $6.14 * * *$ & 69.77 & $0.97 *$ & 98.33 & $-3.27 * * *$ & 71.78 & $-0.47^{*}$ & 13.53 \\
\hline KSP-2*sonali-10 & $-0.15 * * *$ & 0.91 & $6.71 * * *$ & 70.83 & $2.63 * * *$ & 99.00 & $3.10^{* * *}$ & 75.73 & $-0.78 * * *$ & 13.79 \\
\hline
\end{tabular}

Significant at $5 \%$ level; $* *$ significant at $1 \%$ level 
Table.5 Estimation of heterosis over better parent for 10 characters in a 10 parents Half-diallel crosses in pea

\begin{tabular}{|c|c|c|c|c|c|c|c|c|c|c|}
\hline \multirow[t]{2}{*}{ Cross combination } & \multicolumn{2}{|c|}{$\begin{array}{l}\text { Days to } 75 \% \\
\text { Flowering }\end{array}$} & \multicolumn{2}{|c|}{ Plant height $(\mathrm{cm})$} & \multicolumn{2}{|c|}{$\begin{array}{l}\text { Primary } \\
\text { branches/plant }\end{array}$} & \multicolumn{2}{|c|}{$\begin{array}{l}\text { Pod length } \\
(\mathrm{cm})\end{array}$} & \multicolumn{2}{|c|}{ Seeds/pod } \\
\hline & $F_{1 \text { MEAN }}$ & $\mathrm{BH}$ & $\mathrm{F}_{1 \text { MEAN }}$ & $\mathrm{BH}$ & $F_{1 \text { MEAN }}$ & $\mathrm{BH}$ & $\mathrm{F}_{1 \text { MEAN }}$ & $\mathrm{BH}$ & $\mathrm{F}_{1 \text { MEAN }}$ & $\mathrm{BH}$ \\
\hline Pear Polo-5*VRPE-32 & 70.33 & -2.99 & 61.23 & $-12.71^{* *}$ & 4.60 & $21.05^{* *}$ & 9.12 & $12.13 * *$ & 8.43 & $4.12 *$ \\
\hline Pear Polo-5*L-116 & 71.00 & $-9.36 * *$ & 60.41 & $-15.70 * *$ & 5.30 & $72.83^{* *}$ & 8.92 & $2.86^{*}$ & 8.40 & $11.75^{* *}$ \\
\hline Pear Polo-5*AP-3 & 73.33 & $-3.51 *$ & 68.79 & $-5.95^{* *}$ & 4.27 & $26.11 * *$ & 9.34 & $22.40 * *$ & 7.40 & $-9.76 * *$ \\
\hline Pear Polo-5*RACHANA & 71.00 & $-3.40 *$ & 69.34 & $-10.08 * *$ & 3.40 & 3.55 & 8.12 & $3.00 *$ & 9.47 & $16.39 * *$ \\
\hline Pear Polo-5*VRPE-24 & 69.33 & $-9.17 * *$ & 70.19 & $-4.02 * *$ & 4.13 & $29.84 * *$ & 7.87 & $-11.50^{* *}$ & 9.53 & $18.43^{* *}$ \\
\hline Pear Polo-5*JYOTI & 68.67 & $-9.85^{* *}$ & 71.68 & $7.23 * *$ & 5.37 & $88.30^{* *}$ & 8.46 & 4.38 & 7.03 & $-12.99 * *$ \\
\hline Pear Polo-5*PB-01 & 69.67 & $-5.43 * *$ & 75.87 & $-0.85 * *$ & 4.33 & $30.65 * *$ & 9.13 & $4.94 *$ & 8.20 & 1.44 \\
\hline Pear Polo-5*KSP-2 & 70.33 & $-5.38 * *$ & 70.66 & $-3.18 * *$ & 4.83 & $23.40 * *$ & 7.77 & 1.59 & 7.33 & $-5.78 * *$ \\
\hline Pear Polo-5*sonali-10 & 72.00 & $-5.68 * *$ & 68.88 & $-10.39 * *$ & 3.90 & $6.36 * *$ & 8.67 & $-3.11 *$ & 8.70 & $21.11 * *$ \\
\hline VRPE-32*L-116 & 72.00 & $-8.28 * *$ & 73.74 & $4.85 * *$ & 4.53 & $27.10 * *$ & 9.02 & $8.96 * *$ & 6.70 & $-15.72 * *$ \\
\hline VRPE-32*AP-3 & 72.67 & $-4.60 * *$ & 71.34 & $-0.67 *$ & 4.73 & $21.89 * *$ & 8.25 & $13.94 * *$ & 8.40 & -2.70 \\
\hline VRPE-32*RACHANA & 71.67 & -2.71 & 65.87 & $-13.09 * *$ & 3.63 & -3.96 & 8.58 & $14.55^{* *}$ & 7.53 & $-12.06^{* * *}$ \\
\hline VRPE-32*VRPE-24 & 72.67 & $-5.01 * *$ & 68.47 & $-4.65 * *$ & 4.53 & $23.08 * *$ & 8.60 & 1.10 & 8.37 & -1.38 \\
\hline VRPE-32*JYOTI & 72.33 & $-5.24 * *$ & 70.12 & $7.02 * *$ & 3.27 & -2.49 & 7.37 & $-4.47 * *$ & 7.93 & $-6.85^{* *}$ \\
\hline VRPE-32*PB-01 & 72.33 & -2.03 & 64.63 & $-14.05 * *$ & 4.83 & $26.64 * *$ & 8.89 & $7.06^{* *}$ & 8.80 & 3.33 \\
\hline VRPE-32*KSP-2 & 71.33 & $-4.25 * *$ & 69.88 & $-2.49 * *$ & 4.43 & 0.38 & 7.85 & $8.20 * *$ & 7.47 & $-9.13 * *$ \\
\hline VRPE-32*sonali-10 & 69.00 & $-9.80 * *$ & 70.34 & $-6.89 * *$ & 3.43 & $-17.60 * *$ & 8.38 & -2.12 & 8.47 & $11.16^{* *}$ \\
\hline L-116*AP-3 & 70.00 & $-14.63 * *$ & 67.45 & $-8.02 * *$ & 5.10 & $61.90 * *$ & 7.47 & $-4.01 * *$ & 6.63 & $-17.60 * *$ \\
\hline L-116*RACHANA & 69.00 & $-13.21 * *$ & 63.24 & $-18.20 * *$ & 2.87 & $-6.01 *$ & 8.46 & $5.38 * *$ & 9.13 & $14.41 * *$ \\
\hline L-116*VRPE-24 & 69.33 & $-15.79 * *$ & 71.44 & $-2.56^{* *}$ & 3.77 & $27.68 * *$ & 8.12 & $-10.16^{* *}$ & 8.37 & $5.91 * *$ \\
\hline L-116*JYOTI & 68.67 & $-16.43 * *$ & 70.29 & $4.87 * *$ & 4.73 & $80.89 * *$ & 7.63 & $-7.47 * *$ & 7.53 & $-5.04 *$ \\
\hline L-116*PB-01 & 70.33 & $-11.72 * *$ & 69.39 & $-9.54 * *$ & 3.83 & $24.32 * *$ & 8.93 & 1.00 & 8.30 & $4.62 *$ \\
\hline L-116*KSP-2 & 72.67 & $-9.54 * *$ & 63.86 & $-12.73 * *$ & 4.27 & $15.84 * *$ & 9.11 & $16.94 * *$ & 6.63 & $-13.10^{* *}$ \\
\hline L-116*sonali-10 & 69.33 & $-15.79 * *$ & 67.83 & $-11.98 * *$ & 5.33 & $55.34 * *$ & 7.85 & $-13.74 * *$ & 8.70 & $23.70 * *$ \\
\hline AP-3*RACHANA & 71.33 & $-7.56^{* *}$ & 72.64 & $-7.80 * *$ & 2.80 & $-16.83 * *$ & 8.55 & $22.42 * *$ & 8.00 & $-7.69 * *$ \\
\hline AP-3*VRPE-24 & 69.00 & $-13.75 * *$ & 65.83 & $-12.00 * *$ & 4.57 & $39.80 * *$ & 7.92 & -1.08 & 6.60 & $-23.11 * *$ \\
\hline AP-3*JYOTI & 70.33 & $-11.90 * *$ & 68.88 & 0.53 & 3.17 & $7.95 * *$ & 8.33 & $15.51 * *$ & 7.67 & $-11.03 * *$ \\
\hline AP-3*PB-01 & 70.33 & $-9.05^{* *}$ & 63.74 & $-18.48 * *$ & 4.60 & $35.29 * *$ & 7.65 & -1.96 & 9.07 & $5.22 * *$ \\
\hline AP-3*KSP-2 & 71.67 & $-8.12 * *$ & 69.37 & $-7.08 * *$ & 4.77 & $19.17 * *$ & 7.88 & $16.70^{* *}$ & 6.70 & $-19.44 * *$ \\
\hline AP-3*sonali-10 & 70.33 & $-12.08 * *$ & 71.37 & $-9.13 * *$ & 2.47 & $-34.22 * *$ & 8.12 & 0.81 & 7.60 & -1.51 \\
\hline RACHANA*VRPE-24 & 71.33 & $-7.96^{* *}$ & 65.56 & $-16.78 * *$ & 3.70 & $16.84 * *$ & 8.32 & 0.89 & 8.37 & -1.76 \\
\hline RACHANA*JYOTI & 71.33 & $-7.76^{* *}$ & 69.45 & $-4.19 * *$ & 2.70 & -4.71 & 7.56 & 1.41 & 6.77 & $-20.86^{* * *}$ \\
\hline RACHANA*PB-01 & 70.33 & $-6.01 * *$ & 69.67 & $-15.21 * *$ & 3.87 & $17.17 * *$ & 7.90 & -1.82 & 8.53 & -0.19 \\
\hline RACHANA*KSP-2 & 70.00 & $-7.28 * *$ & 72.64 & $-7.62 * *$ & 4.70 & $20.51 * *$ & 8.42 & $20.26 * *$ & 8.50 & 3.03 \\
\hline RACHANA*sonali-10 & 70.67 & $-8.82 * *$ & 64.84 & $-21.42 * *$ & 3.53 & -3.20 & 7.73 & $-6.92 * *$ & 8.73 & $14.16^{* *}$ \\
\hline VRPE-24*JYOTI & 71.67 & $-10.60 * *$ & 70.25 & $2.54 * *$ & 2.83 & 3.66 & 8.43 & -0.49 & 6.67 & $-21.26 * *$ \\
\hline VRPE-24*PB-01 & 71.67 & $-7.73 * *$ & 70.62 & $-9.68 * *$ & 4.23 & $32.29 * *$ & 6.97 & $-23.16^{* *}$ & 8.27 & -2.36 \\
\hline VRPE-24*KSP-2 & 71.33 & $-8.94 * *$ & 71.75 & $-3.88 * *$ & 3.47 & $-8.77 * *$ & 7.67 & $-4.39 * *$ & 9.03 & $10.61 * *$ \\
\hline VRPE-24*sonali-10 & 71.67 & $-10.79 * *$ & 69.53 & $-11.46^{* *}$ & 4.83 & $36.15^{* *}$ & 8.44 & $-9.42 * *$ & 7.50 & -0.88 \\
\hline JYOTI*PB-01 & 70.00 & $-9.68 * *$ & 66.57 & $-7.40 * *$ & 4.17 & $45.35 * *$ & 8.18 & -1.19 & 9.07 & $6.67 * *$ \\
\hline JYOTI*KSP-2 & 73.00 & $-6.61 * *$ & 69.02 & $0.96 * *$ & 3.30 & $-4.81 *$ & 7.58 & $4.89 * *$ & 6.80 & $-17.07 * *$ \\
\hline JYOTI*sonali-10 & 71.67 & $-10.60 * *$ & 73.66 & $1.96 * *$ & 2.83 & $-11.92 * *$ & 8.43 & -1.17 & 8.47 & $11.40 * *$ \\
\hline PB-01*KSP-2 & 70.33 & $-7.05^{* *}$ & 63.48 & $-18.66 * *$ & 3.83 & -2.54 & 8.30 & $6.16^{* *}$ & 7.00 & $-14.63 * *$ \\
\hline PB-01*sonali-10 & 72.33 & $8.87 * *$ & 65.39 & $-20.18 * *$ & 4.77 & 29.41 & 7.76 & $-14.96 * *$ & 8.73 & $14.9 * *$ \\
\hline KSP-2*sonali-10 & 72.00 & $-8.09 * *$ & 66.71 & $-14.90 * *$ & 4.67 & $8.95 * *$ & 6.82 & $-15.52 * *$ & 7.43 & 1.83 \\
\hline
\end{tabular}

Significant at $5 \%$ level; $* *$ significant at $1 \%$ level 
Table.5 Contd.

\begin{tabular}{|c|c|c|c|c|c|c|c|c|c|c|}
\hline \multirow[t]{2}{*}{ Cross combination } & \multicolumn{2}{|c|}{ Pod density $(\mathrm{cm})$} & \multicolumn{2}{|l|}{ Pods/plant } & \multicolumn{2}{|c|}{ Days to maturity } & \multicolumn{2}{|c|}{ Yield/ Plant $\mathrm{g}(\mathrm{kg})$} & \multicolumn{2}{|c|}{ 100-seed weight $(\mathrm{g})$} \\
\hline & $F_{1 \text { MEAN }}$ & $\mathrm{BH}$ & $\mathrm{F}_{1 \text { MEAN }}$ & $\mathrm{BH}$ & $\mathrm{F}_{1 \text { MEAN }}$ & $\mathrm{BH}$ & $\mathrm{F}_{1 \text { MEAN }}$ & $\mathrm{BH}$ & $\mathrm{F}_{1 \mathrm{MEAN}}$ & $\mathrm{BH}$ \\
\hline Pear Polo-5*VRPE-32 & 1.08 & 7.13 & 55.41 & 1.73 & 92.67 & $-3.81 * *$ & 66.57 & $-9.49^{* *}$ & 12.85 & -0.10 \\
\hline Pear Polo-5*L-116 & 1.06 & $-7.98 *$ & 56.66 & $10.42 * *$ & 95.67 & $-2.71 * *$ & 70.66 & -0.26 & 13.86 & 0.48 \\
\hline Pear Polo-5*AP-3 & 1.26 & $34.76^{* * *}$ & 57.48 & $14.67 * *$ & 94.00 & -0.88 & 72.87 & $-1.78^{* *}$ & 14.44 & $17.09^{* *}$ \\
\hline Pear Polo-5*RACHANA & 0.85 & $-12.18^{* *}$ & 57.35 & $7.06^{* * *}$ & 95.33 & $-4.35 * *$ & 68.05 & $-9.13^{* *}$ & 13.86 & -2.52 \\
\hline Pear Polo-5*VRPE-24 & 0.82 & $-25.45 * *$ & 59.98 & $9.80^{* * *}$ & 93.00 & $-3.63 * *$ & 69.49 & $-3.45^{* *}$ & 13.64 & $7.51^{* *}$ \\
\hline Pear Polo-5*JYOTI & 1.20 & $19.80 * *$ & 60.74 & $13.72 * *$ & 98.00 & -1.18 & 73.60 & $-3.11 * *$ & 12.62 & $-4.96^{* * *}$ \\
\hline Pear Polo-5*PB-01 & 1.11 & 3.58 & 58.62 & $7.70^{* *}$ & 97.00 & $-2.51 * *$ & 74.45 & $-2.94 * *$ & 13.79 & $12.84^{* *}$ \\
\hline Pear Polo-5*KSP-2 & 1.05 & $7.48 *$ & 59.97 & $7.60 * *$ & 101.33 & 5.19 & 66.60 & $-10.73 * *$ & 13.72 & -0.06 \\
\hline Pear Polo-5*sonali-10 & 0.99 & $-20.91 * *$ & 59.78 & $9.00 *$ & 102.00 & 9.09 & 75.78 & $4.40 * *$ & 12.64 & $-10.94 * *$ \\
\hline VRPE-32*L-116 & 1.35 & $28.25 * *$ & 60.68 & $13.87^{* * *}$ & 94.33 & $-5.35 * *$ & 68.78 & $-5.92 * *$ & 11.63 & $-16.67 * *$ \\
\hline VRPE-32*AP-3 & 1.00 & $19.12^{* *}$ & 61.65 & $18.33^{* *}$ & 96.67 & 0.52 & 67.57 & $-11.63 * *$ & 14.62 & $17.03 * *$ \\
\hline VRPE-32*RACHANA & 1.14 & $30.15 * *$ & 61.55 & $10.79^{* *}$ & 102.00 & 0.99 & 76.71 & -0.57 & 13.68 & $-4.88 * *$ \\
\hline VRPE-32*VRPE-24 & 1.02 & 2.16 & 60.64 & $7.12 * *$ & 98.33 & 0.51 & 66.04 & $-11.06 * *$ & 15.28 & $18.94 * *$ \\
\hline VRPE-32*JYOTI & 0.92 & 2.21 & 61.51 & $11.04 * *$ & 96.00 & $-4.48 * *$ & 65.43 & $-16.36^{* *}$ & 14.41 & $7.23^{* *}$ \\
\hline VRPE-32*PB-01 & 1.01 & 3.77 & 55.66 & -1.33 & 94.33 & $-6.45 * *$ & 71.19 & $-9.85 * *$ & 13.42 & $8.44 * *$ \\
\hline VRPE-32*KSP-2 & 1.05 & $18.71 * *$ & 57.76 & 0.08 & 96.67 & -1.02 & 73.38 & $-4.55^{* *}$ & 12.68 & $-8.65^{* *}$ \\
\hline VRPE-32*sonali-10 & 0.98 & $-14.74 * *$ & 60.71 & $6.85 * *$ & 91.33 & $-3.69 * *$ & 74.60 & -0.35 & 15.55 & $8.34 * *$ \\
\hline L-116*AP-3 & 1.12 & $14.63 * *$ & 61.62 & $25.91 * *$ & 95.33 & $-2.89 * *$ & 65.38 & $-11.35^{* *}$ & 14.65 & $9.14 * *$ \\
\hline L-116*RACHANA & 0.92 & $-9.51 * *$ & 63.99 & $22.13^{* * *}$ & 103.67 & 0.65 & 67.48 & $-9.35 * *$ & 14.71 & $-3.97 *$ \\
\hline L-116*VRPE-24 & 0.96 & $-15.87 * *$ & 64.68 & $21.01 * *$ & 97.33 & $-2.50 * *$ & 71.30 & -0.32 & 11.66 & $-15.38 * *$ \\
\hline L-116*JYOTI & 1.01 & -3.82 & 65.56 & $25.52 * *$ & 95.67 & $-6.67 * *$ & 68.49 & $-9.31 * *$ & 13.65 & $-5.07 * *$ \\
\hline L-116*PB-01 & 1.07 & -4.18 & 58.41 & $9.70 * *$ & 99.00 & $-3.73 * *$ & 74.70 & $-2.05 * *$ & 12.63 & $-5.14 * *$ \\
\hline L-116*KSP-2 & 1.37 & $33.98 * *$ & 59.86 & $9.74 * *$ & 97.00 & $-2.68 * *$ & 71.38 & $-3.76^{* * *}$ & 11.68 & $-21.17 * *$ \\
\hline L-116*sonali-10 & 0.90 & $-30.59 * *$ & 60.05 & $11.90^{* *}$ & 96.00 & -0.86 & 65.80 & $-8.80 * *$ & 15.77 & 3.14 \\
\hline AP-3*RACHANA & 1.08 & $34.44 * *$ & 62.29 & $21.64 * *$ & 102.33 & 2.85 & 63.61 & $-18.22 * *$ & 12.40 & $-10.48 * *$ \\
\hline AP-3*VRPE-24 & 1.03 & $10.55 * *$ & 62.67 & $19.91 * *$ & 95.33 & -1.04 & 68.86 & $-8.04 * *$ & 11.46 & $-6.93 * *$ \\
\hline AP-3*JYOTI & 1.08 & $29.60 * *$ & 63.84 & $25.06^{* * *}$ & 94.33 & $-4.71 * *$ & 70.59 & $-10.50 * *$ & 14.43 & $11.79^{* * *}$ \\
\hline AP-3*PB-01 & 0.84 & -7.01 & 55.90 & $7.38 * *$ & 97.33 & $-2.01 * *$ & 71.62 & $-10.03 * *$ & 11.65 & -1.65 \\
\hline AP-3*KSP-2 & 1.17 & $44.56^{* *}$ & 61.54 & $15.31 * *$ & 98.00 & 1.91 & 74.44 & $-3.97 * *$ & 13.74 & 2.88 \\
\hline AP-3*sonali-10 & 1.06 & -1.85 & 56.87 & $8.39 * *$ & 101.00 & 8.21 & 78.89 & $4.49 * *$ & 15.08 & $9.04 * *$ \\
\hline RACHANA*VRPE-24 & 0.99 & 2.24 & 53.37 & $-4.21 * *$ & 100.33 & -0.82 & 67.87 & $-10.19 * *$ & 15.38 & $8.25 * *$ \\
\hline RACHANA*JYOTI & 1.11 & $27.97 * *$ & 61.45 & $12.75 * *$ & 96.00 & $-7.54 * *$ & 62.01 & $-22.06 * *$ & 11.54 & $-22.07 * *$ \\
\hline RACHANA*PB-01 & 0.92 & -1.77 & 65.60 & $18.18 * *$ & 97.67 & $-6.24 * *$ & 65.69 & $-18.20 * *$ & 15.25 & $11.01 * *$ \\
\hline RACHANA*KSP-2 & 0.99 & $16.31 * *$ & 58.82 & $3.52 * *$ & 96.00 & $-4.95 * *$ & 65.12 & $-16.73 * *$ & 14.46 & $-5.14 * *$ \\
\hline RACHANA*sonali-10 & 0.88 & $-21.13 * *$ & 58.41 & $4.45^{* *}$ & 96.00 & $-2.21 * *$ & 69.47 & $-8.82 * *$ & 12.32 & $-21.62 * *$ \\
\hline VRPE-24*JYOTI & 1.26 & $26.21 * *$ & 69.53 & $25.16^{* *}$ & 96.00 & $-4.64 * *$ & 75.83 & $-1.08^{*}$ & 14.64 & $10.36^{* *}$ \\
\hline VRPE-24*PB-01 & 0.84 & $-21.68 * *$ & 71.76 & $26.85^{* * *}$ & 94.33 & $-6.60 * *$ & 79.38 & $2.56^{* *}$ & 13.64 & $11.76^{* *}$ \\
\hline VRPE-24*KSP-2 & 0.85 & $-13.31 * *$ & 70.61 & $22.01 * *$ & 94.67 & $-3.24 * *$ & 65.76 & $-12.67 * *$ & 14.23 & $3.76^{*}$ \\
\hline VRPE-24*sonali-10 & 1.12 & $-10.28 * *$ & 56.90 & -0.15 & 97.00 & 2.11 & 64.34 & $-12.20 * *$ & 15.25 & $7.54 * *$ \\
\hline JYOTI*PB-01 & 0.89 & $-7.90^{*}$ & 71.82 & $29.75^{* *}$ & 100.00 & $-3.54 * *$ & 72.01 & $-11.52 * *$ & 13.70 & $7.01 * *$ \\
\hline JYOTI*KSP-2 & 1.11 & $26.38 * *$ & 68.76 & $21.36^{* * *}$ & 96.00 & $-4.48 * *$ & 67.72 & $-14.59 * *$ & 13.98 & -2.28 \\
\hline JYOTI*sonali-10 & 1.15 & -0.29 & 70.77 & $26.90 * *$ & 95.67 & $-2.05 * *$ & 70.65 & $-8.57 * *$ & 14.69 & -0.62 \\
\hline PB-01*KSP-2 & 1.18 & $24.43 * *$ & 71.81 & $24.52 * *$ & 95.00 & $-5.79 * *$ & 73.75 & $-7.84 * *$ & 14.09 & $6.42 * *$ \\
\hline PB-01*sonali-10 & 0.88 & $-27.80 * *$ & 69.77 & $2.88 * *$ & 98.33 & 0.34 & 71.78 & $-7.99 * *$ & 13.53 & -1.32 \\
\hline KSP-2*sonali-10 & 0.91 & $-19.35^{* *}$ & 70.83 & $21.94 * *$ & 99.00 & 4.39 & 75.73 & -0.25 & 13.79 & $-9.43^{* *}$ \\
\hline
\end{tabular}

Significant at $5 \%$ level; $* *$ significant at $1 \%$ level

\section{General combining ability (GCA) effects}

The estimated of general combining ability effects of ten parents for all the 10 characters in $F_{1}$ generations along with their per se performance are given in Table 3 . The lowest negative and significant values of GCA effects were considered to find out the desirable for number of days to $75 \%$ flowering. Plant height, days to maturity while for remaining characters have positive and significant GCA effects were taken into account for sorting out the desirable general combiners. Similar, justification was also reported by Enrique Luis Cointry et al., (2013) in which significant results for GCA and SCA for plant height was obtained. In $\mathrm{F}_{1}$ generation, significant and desirable GCA effect were observed for days to 75\% flowering in Pearpol-5,VRPE-32, Rachana 
andPB-01,KSP-2; for plant height in Pearpolo-5, VRPE-32L-116, Jyoti and KSP-2; for days to maturity in Sonali-10, AP3,Pearpolo-5,VRPE-32 and KSP-2. The parents observed desirable and significant general combiners in $F_{1}$ the generation for days to $75 \%$ flowering was Pearpolo5,Rachana,PB-01, VRPE-32; for plant height were L-116, VRPE-32, Peapolo-5, Jyoti; for primary branches per plant were KSP2,Pearpolo-5,VRPE-32, and PB-01; for pod length were Pearpolo-5, L-116, VRPE-32PB01 and Sonali-10; for seeds per pod PB-01, Rachana, Pearpolo-5 and VRPE-24; for pod density L-116,Sonali-10,Pearpolo-5 and Jyoti; for pods per plant Jyoti, KSP-2, PB-01, and VRPE-24; for days to maturity VRPE-32, Pearpolo-5, Sonali-10, VRPE-24, KSP-2; for yield per plant PB-01, KSP-2, Sonali -10, AP3; for 100- seed weight Sonali-10, Rachana, KSP-2, Jyoti. The study revealed significant difference in GCA and SCA effects for the traits viz., days to $75 \%$ flowering, number of primary branches per plant, pod per plant, days to maturity and seed yield per plant. These findings were supported by the previous work of Singh et al., (2001) and Dixit et al., (2003). Based on desirable GCA effect for different characters, the per se performance and best common general combiner in F1 were identified for days to $75 \%$ flowering was PB-01,Pearpolo-5, Rachana, VRPE-32; for plant height were L116, VRPE-32, Pearpolo-5 and Jyoti; for primary branches per plant were KSP-2, VRPE-32, PB-01; for pod length per plant and seeds per pods both were Pearpolo-5, VRPE-32, L-116, PB-01, Sonali-10 and Rachana,PB-01, Pearpolo-5 VRPE-24; for yield per plant.

\section{Specific combining ability (SCA) effect}

The specific combining ability (SCA) effects of 45 crosses in $F_{1} s$ along with the mean performance for 10 characters are given in
Table 4. The significant specific combiners and desirable crosses were screened out same as in case of (SCA) effects. The significant and desirable (SCA) effects in $\mathrm{F}_{1}$ generation were found in 35 combinations for days to 75 percent flowering showed negative value. Out of these, four best specific combiners with high per se performance were VRPE-24/KSP2, Pearplol-5/VRPE-24, L-116/Rachana, Pearpolo-5/Rachana-116/Sonali-10; in F1 L116/Jyoti, L-116/PB-01, AP-3/VRPE-24, L116/Sonali-10, VRPE-32/Sonali-10. Plant height exhibited 26 significant and desirable combinations. Out of these five combinations like Pearpolo-5/VRPE-32, Pearpolo-5/L-116, PB-01/KSP-2，AP-3/PB-01， L-116/Rachana showed high per se performance but good specific combiner in $F_{1}$ were identified i.e. Pearpolo-5/L-116, Rachana/Sonali-10, Pearpolo-5/VRPE-32, L-116/Rachana. In $\mathrm{F}_{1}$ days to maturity 27 combinations showed negative significant and desirable sca effect. On the basis of per se performance five crosses were found desirable i.e. Pearpolo5/VRPE-32, VRPE-32/Sonali-10, Pearpolo5/Jyoti, Pearpolo-5/VRPE-24, PB-01/KSP-2 in $F_{1}$ were good specific combiners.

Considering the $F_{1}$ generations simultaneously, superior common crosses for the days to $75 \%$ flowering was L-116/Sonali10 based on sca and per se performance. For plant height PB-01/KSP-2, L-116/Rachana, Pearpolo-5/VRPE-32; for Primary branches per plant Pearpolo-5/Jyoti; for Pod length L116/KSP-2 and Pearpolo-5/AP-3; for Seeds per pod Pearpolo-5/Rachana, Pearpolo5/VRPE-32; for Pod density L-116/KSP2,VRPE-24/Jyoti, Pearpolo-5/AP-3, VRPE32/L-116; for Pods per plant were Jyoti/Sonali-10, PB-01/KSP-2, VRPE-24/PB01, KSP-2/Sonali-10; for Days to maturity VRPE-32/Sonali-10; for Yield per plant were VRPE-24/Jyoti and AP-3/KSP-2; for 100 seed weight Rachana/PB-01 and VRPE-32/VRPE24 ,were most desirable and common cross 
combinations based on sca and per se performance.

Considerable degree of heterosis was noted for grain yield in positive response over better parent namely, Pearpolo-5/Jyoti and Pearpolo-5/AP-3.The relative ranking of the parents on the basis of per se performance and GCA effect in $\mathrm{F}_{1}$ generations Jyoti, Pearpol-5, Rachana, Sonali-10 and AP-3 are good combiner for yield point of view. These parents could be exploited further in breeding programme for simultaneously improved in grain yield and quality attributes. The SCA effect for grain yield per plant was significant and considerable good specific combiner were four crosses in F1 generations. These crosses had involved all the four possible combination between high and low GCA effects. The cross combinations involving parents Pearpolo-5, KSP-2, PB-01 and VRPE32 were expected to have fixable, additive or additive $\mathrm{x}$ additive gene effects.

\section{Acknowledgement}

We are extremely thanks to Department of Genetics and Plant Breeding, School of Agriculture, Lovely Professional University, Phagwara (Punjab), for valuable suggestions and assistance provided during the course of investigation.

\section{References}

Acikgoz E., Ustun A., Gul I., Anlarsal E., Tekeli A.S., Nizam I., Avcioglu R., Geren H., Cakmakci S., Aydinoglu B., Yucel C., Avci M., Acar Z., Ayan I., Uzun A., Bilgili U., Sincik M., Yavuz M. (2009) Genotype x environment interaction and stability analysis for dry matter and seed yield in field pea (Pisum sativum L.). Spanish Journal of Agricultural Research 7:96-106.
Tyagi, M.K and Srivastava, C.P. (2001). Analysis of gene effects in pea. Legume Research., 24(2): 71-76.

Swaminathan, M.S., (2002) Food groups and balance diet: Recommended dietary allowances. In: Essentials of Food and Nutrition - An Advanced Textbook, Vol. 2. pp. 1-23. Bangalore: The Bangalore Printing and Publishing Co. Ltd.

Sharma, A.C, Singh. S.P. and Sharma, M.K.(2003).Genetic variability, genetic ability and character association in pea (Pisum sativum L.).Res. 26(1): 135-139.

Alam, A.K.M.M., Ahmed, S., Begum, M and Sultan, M.K. 2008. Heterosis and combining ability for grain yield and its contributing characters in maize. Bangladesh Journal of Agricultural Research. 33(3): 375-379.

Ceyhan E (2003). Determination of some agricultural characters and their heredity through line $\mathrm{x}$ tester method in pea parents and crosses. Selcuk Univ., Graduate School Nat. Appl. Sci. p. 103

Mehmet al.,i and Avci (2008). Line x tester analysis in pea (Pisum sativum L.). Identification of superior parents for seed yield and its components. African. J. Bio., 7(16): 2810-2817

Mohammad Motamedi, Rajab choukan, Eslam majidi hervan, Mohammad Reza Bihamta and Farokh Darvish Kajouri. (2014) Int. J. Biosci. Vol. 5, No. 12, p. 123-129.

Griffing B (1956b). Concept of general and specific combining ability in relation to diallel crossing system. Aust. J. Biol. Sci. 9:463-493.

Dixit GP (2003). Combining ability analysis in Field pea. Indian J. Pulses Res. 16(1):20-21.

Singh NK, Kumar D, Kumar N, Singh DN (2001). Combining ability for yield 
and its components in pea. Ann. Agric. Res. 22(4):570-575.

Enrique Luis Cointry, María Andrea Espósito, leana Gatti and Fernando Sebastián López Anido1,(2013). Combining abilities and heterotic groups in Pisum sativum L. Australian Journal of Crop Science : 7(11):1634-1641.

Kempthorne O (1957). An Introduction of Genetics Statistics. John Wiley and Sons, New York. pp. 458-471.

Sing, R.K. and B.D. Chaudhary, 1979. Line Tester Analysis Biometrical Methods in Quantitative Genetic Analysis. Kalyani Publishers, New Delhi, India.

Hayman, B.I., 1954. The theory and analysis of diallel crosses. Genetics, 39: 789809.

Singh, K. P., Singh, H. C. and Verma, M. C. 2010. Genetic analysis for yield and yield traits in pea. J. Food Legume. 23 (2) : $113-116$.

Singh, A. and Lavanya, G.R. 2014. Character association studies in field pea (Pisum sativum L.) Trends Biosci., 7 (1): 5153.

\section{How to cite this article:}

Hariom Suman, Bijendra Kumar, Nageshwar, Meenakshi Rathi and Dattesh Tamatam. 2017. Heterosis and Combining Ability for Grain Yield and Yield Associated Traits in 10 X 10 Diallel Analysis in Pea (Pisum sativum L.). Int.J.Curr.Microbiol.App.Sci. 6(12): 1574-1585. doi: https://doi.org/10.20546/ijcmas.2017.612.177 